\title{
Teoría y métodos para la investigación de la racionalidad de la práctica en la enseñanza de las matemáticas
}

\section{Theory and methods for research on the practical rationality of mathematics teaching}

\section{Patricio Herbst ${ }^{1}$}

\begin{abstract}
Resumen: Este artículo sintetiza en castellano un programa de investigación en la racionalidad de la práctica de la enseñanza de las matemáticas, partiendo de la problemática de la toma de decisiones en el salón de clases. Se proveen referencias a artículos escritos en inglés durante los pasados 15 años, que dan cuenta de investigaciones en aspectos específicos de aquella racionalidad. Se definen las nociones de transacción de instrucción, situación de instrucción, norma de una situación, y obligación profesional, y luego se describen instrumentos diseñados para medir el grado en el que los maestros reconocen las normas de instrucción y las obligaciones profesionales. Se ilustra cómo funcionan esos constructos mediante el esbozo de algunas preguntas de investigación.
\end{abstract}

Fecha de recepción: 9 de febrero de 2018. Fecha de aceptación: 9 de marzo de 2018 University of Michigan, Ann Arbor, Estados Unidos de Norteamérica

Patricio Herbst (pgherbst@umich.edu). 610 East University Ave. \#4215B. Ann Arbor, MI 48109-1259. Estados Unidos de Norteamérica

1 Texto revisado de la conferencia plenaria dictada en ocasión del Tercer Coloquio de Doctorado en Matemática Educativa en el CINVESTAV, IPN, Ciudad de México. El autor agradece a Margarita Curiel por su apoyo con la transcripción de la grabación y a Vilma Mesa por comentarios sobre una versión anterior. El desarrollo de las ideas ha surgido del trabajo conjunto con Daniel Chazan desde 2001 y ha contado con el apoyo de la United States National Science Foundation (NSF) mediante subsidios a la investigación REC-0133619, ESI-0353285, y DRL- 0918425. Todas las opiniones expresadas en este ensayo son las del autor y no representan la posición de la National Science Foundation. 
Palabras clave: racionalidad de la práctica, situación de instrucción, norma de instrucción, demostración.

\begin{abstract}
A program of research on the practical rationality of mathematics teaching is introduced in Spanish, starting from problematizing teachers' decision making in instruction. References to specific articles covering 15 years of research on the subject are provided. The notions of instructional exchange, instructional situation, situational norm, and professional obligation are introduced, then instruments designed to measure teachers' recognition of norms and obligations are described, and research questions are proposed.
\end{abstract}

Key words: practical rationality, instructional situation, instructional norm, proof.

¿Cómo se pueden explicar las decisiones que toman los maestros y que conciernen la transacción de conocimientos durante la enseñanza? Por ejemplo, ¿cómo podemos explicar la variación en enunciados de problemas que requieren una demostración en clases de geometría? Utilizo esa última pregunta como ejemplo para anclar conceptos más generales que propongo para examinar la primera pregunta. El enfoque que propongo intenta considerar la especificidad matemática de las transacciones de conocimientos. Pero los constructos propuestos por Herbst y Chazan (2012) son relativamente generales y pueden aplicarse a transacciones de distintos conocimientos matemáticos. En este artículo me refiero a la gestión de las situaciones de demostración en la clase de geometría y a la racionalidad de la práctica que guía tal gestión. Cuando hablo de la racionalidad de la práctica, cuando propongo que para entender la práctica hace falta descubrir cómo se negocian sus normas, esas afirmaciones son metateóricas, sirven para guiar la investigación de teorías locales sobre prácticas específicas, específicas en relación a las transacciones de conocimientos matemáticos específicos.

\title{
EL ESTUDIO DE LA RACIONALIDAD DE LA PRÁCTICA
}

Lo que proponemos al desarrollar elementos para el estudio de la racionalidad de la práctica, es rechazar la tradición prescriptiva con la que la comunidad de investigadores en educación se ha relacionado tradicionalmente 
con el trabajo del maestro. Si bien los investigadores en educación matemática no tienen problema en tomar una actitud descriptiva con respecto al pensamiento y el aprendizaje matemático, la actitud no es la misma con respecto a la enseñanza, la cual es frecuentemente abordada con una actitud prescriptiva. En Estados Unidos, en particular, el trabajo del maestro se ha tratado tradicionalmente como manipulable a voluntad, como si bastara que el maestro tuviera la voluntad de hacer algo en la clase para que tal o cual cosa fuera posible de hacer. Este voluntarismo se ha perfeccionado con la atención a conceptos exclusivamente individualistas: tales como, creencias, conocimientos, e identidad de los maestros para establecer la noción de que a los maestros hay que persuadirlos y capacitarlos para que quieran y sepan hacer lo que deben de hacer. Quienes escriben las políticas educativas frecuentemente deciden lo que los maestros deben de hacer, esbozando visiones y estándares que si bien están informados por resultados de investigación en los aprendizajes, rara vez están informados por el análisis de la complejidad del trabajo del enseñante. Si bien, no nos queda otro recurso que aceptar que los organismos gubernamentales piensen que es posible modificar la enseñanza mediante prescripciones, como investigadores tenemos que tomar una actitud más empírica y reflexiva y estudiar las condiciones de posibilidad de tales cambios.

La perspectiva teórica que atiende a la racionalidad de la práctica mira críticamente a aquella tradición prescriptiva, considerando que la enseñanza ocurre en contextos institucionales que incluyen no solamente políticas educativas y prescripciones explícitas, sino también culturas de trabajo que estimulan y a la vez restringen la instrucción. Cuando digo instrucción me refiero al sistema de interacciones entre el estudiante, el contenido a aprender, y el maestro; a la colección de relaciones mutuas que permiten la transacción de conocimientos. La instrucción, como nos recuerdan tanto Chevallard (1992) como Cohen, Raudenbush, y Ball (2003), ocurre en el contexto de espacios institucionales que la hacen posible a la vez que la sujeta a la influencia de otros agentes y sistemas. En la medida que esas influencias son sociales y tecnológicas (en un sentido amplio), me refiero a ellas como influencias sociotécnicas. El mejoramiento de la enseñanza requiere mucho más que ejercitar la imaginación para diseñar reformas y luego estimular la voluntad e incrementar la capacidad individual de los maestros. El mejoramiento de la enseñanza requiere estudiar la práctica y descubrir las condiciones de posibilidad que pueden facilitar su transformación sistémica. 


\section{DECISIONES EN LA TRANSACCIÓN DE CONOCIMIENTOS}

Desde el punto de vista de la necesidad de entender la práctica podemos ahora volver a la pregunta: ¿Cómo se pueden describir y explicar las decisiones que toman los maestros en las clases y que conciernen la transacción de conocimientos? La pregunta nos invita no a decidir qué es lo que el maestro debería de hacer, sino a estudiar cuán probable es que lo haga. Más aún, la pregunta nos invita a desarrollar elementos teóricos y metodológicos para describir y explicar la variabilidad de las acciones posibles del maestro en clase. El ejemplo en la Figura 1 sirve para anclar el problema; la imagen es parte de un instrumento que describo en detalle más adelante. El maestro presenta el problema en el que quiere que sus estudiantes trabajen.

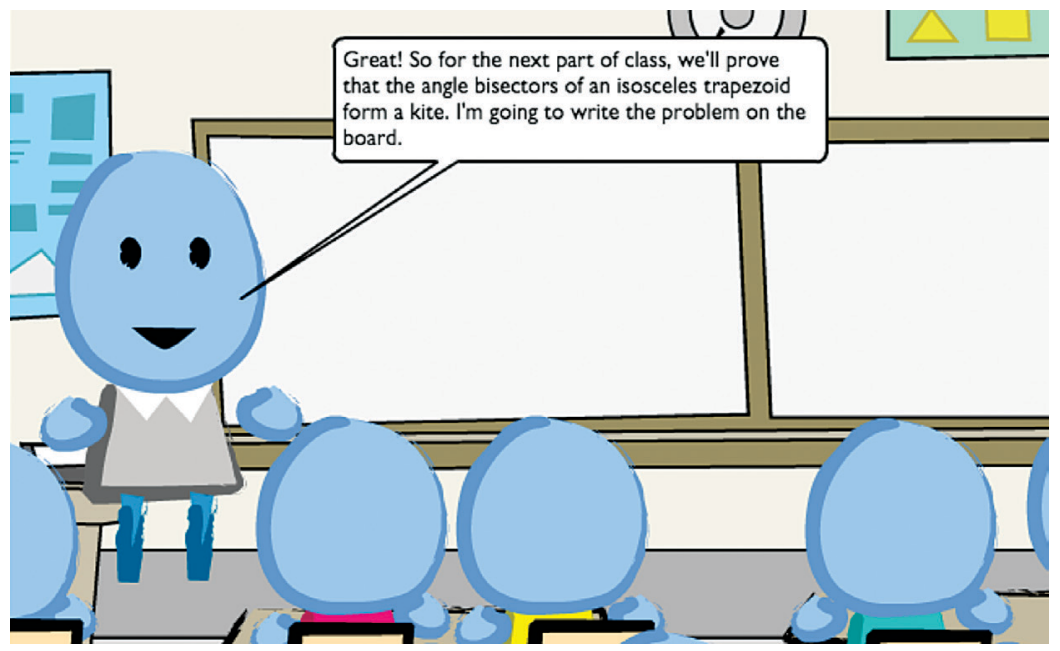

Figura 1. Introduciendo un problema de demostración.²

(C) 2018, The Regents of the University of Michigan, used with permission.

Ante lo presentado en la Figura 1, ¿qué se esperaría que el maestro escriba en el tablero? En la Figura 2 se muestran dos posibilidades; en la Figura 2a, el maestro dice "Copien el diagrama que está en el tablero y trabajen con sus vecinos en el problema;" el enunciado en el tablero dice "¿Por qué podríamos

2 Traducción: "Muy bien; ahora vamos a probar que las bisectrices de los ángulos de un trapecio isósceles forman un romboide. Voy a escribir el problema en el tablero." 
decir que los puntos de intersección de las bisectrices de un trapecio isósceles forman un romboide?" En la Figura 2b el maestro dice algo similar, "Copien el diagrama del tablero y trabajen con sus vecinos para enunciar los datos dados y hacer la prueba." En el tablero está el enunciado que tienen que probar, el cual dice: "Demuestre que los puntos de intersección de las bisectrices de un trapecio isósceles forman un romboide." ¿Cuál acción (2a o 2b) es más probable como continuación de lo mostrado en Figura 1? Y ¿por qué? ¿Qué otras opciones hay y cuáles son sus probabilidades? ¿Qué es lo que está involucrado en las respuestas a estas preguntas?

\section{LA RACIONALIDAD DE LA PRÁCTICA Y LA TOMA DE DECISIONES}

Las perspectivas usuales en investigación en educación y en educación matemática, tratan de reducir la cuestión de la toma de decisiones tanto en términos de cuál es la decisión a tomar, como en términos de cómo explicarla. En términos de cuál decisión tomar, la tarea se suele reducir a decidir sobre la ejecución de un estilo general de enseñanza: por ejemplo decidir entre enseñar por descubrimiento o por instrucción directa. En términos de las causas de la decisión, las explicaciones usuales reducen aquélla a características totalizantes del individuo, como las creencias del maestro: por ejemplo, ¿cree el maestro que los estudiantes pueden hacer problemas difíciles o que los estudiantes necesitan ser guiados paso a paso? Estas perspectivas, si bien razonables, no ponen atención a las matemáticas que están en juego en la toma de decisiones o a la posición institucional del maestro. Cabe hacerse las preguntas, chay aspectos de las concepciones matemáticas en juego que se relacionan con lo que el maestro pueda hacer en la clase? ¿cómo podemos darle atención a las demandas de conocimientos específicas de la situación en la que se toma la decisión? Más aún, ¿hay expectativas depositadas en la acción del maestro que sirvan de trasfondo a la decisión que pueda tomar en este momento en particular? Y ¿qué recursos profesionales tiene disponibles el maestro, dada su posición institucional, que le permitan o le ayuden a justificar una u otra acción? 


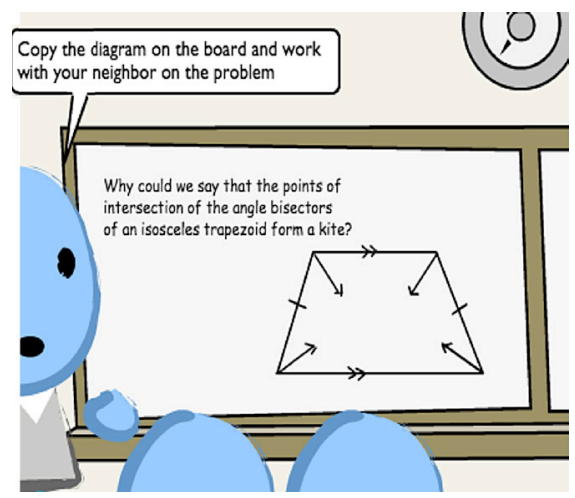

(a)

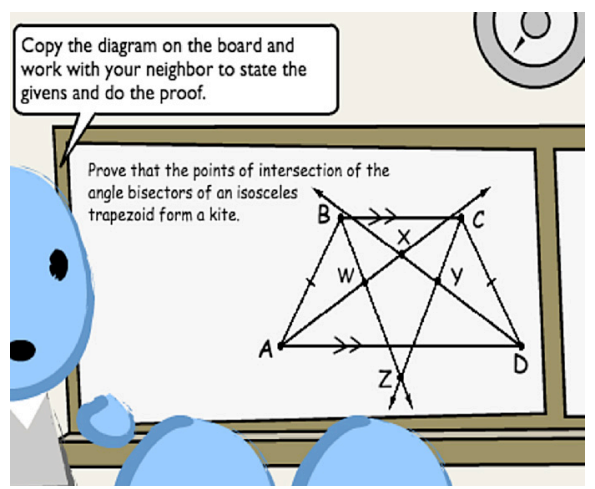

(b)

Figura 2. Dos posibilidades de presentar el problema anunciado en la Figura 1

(c) 2017, The Regents of the University of Michigan y han sido usadas con permiso.

Nuestra perspectiva, que en inglés Ilamamos practical rationality y que traducimos al castellano como racionalidad de la práctica, desarrolla elementos teóricos y metodológicos para estudiar las decisiones del maestro e incluye tres tipos de consideraciones. La primera consideración mantiene la atención vigente en nuestra disciplina al conocimiento personal y otras características individuales del maestro. Las otras dos consideraciones apuntan a dos tipos de recursos sociotécnicos disponibles para el maestro-y al decir sociotécnicos describo aquéllos no solamente como sociales sino también como específicos del conocimiento-. Primeramente, las demandas sociotécnicas de la situación de instrucción, a las que llamo normas de instrucción; en segundo lugar, los recursos sociotécnicos disponibles dada la posición institucional del maestro de matemáticas para justificar decisiones, a las que llamo obligaciones profesionales.

Para identificar los recursos individuales del maestro nos basamos en contribuciones de otros investigadores. Por ejemplo, basados en la teoría del conocimiento matemático de los maestros (MKT) propuesta por Ball, Thames, y Phelps (2008), hemos desarrollado un test que estima el conocimiento de geometría de los maestros (Herbst \& Kosko, 2014a).

Nuestra contribución teórica y metodológica, sin embargo, se centra en constructos y medidas de los recursos sociotécnicos disponibles para el maestro. La perspectiva que tomamos sobre la racionalidad de la práctica acentúa no necesariamente en dónde están esos recursos sociotécnicos, sino 
que describe cuales son los conocimientos de la enseñanza movilizados por las situaciones del trabajo profesional. En particular, este conocimiento puede ser tácito.

Una pregunta que puede surgir al considerar esta tarea es ¿para qué sirve todo esto? La investigación del conocimiento de los enseñantes sirve, por ejemplo, para planificar la formación de docentes. Pero, ¿para qué sirve entender el sistema de instrucción en donde se desempeñan los enseñantes?, ¿para qué puede servir entender los recursos y las restricciones que afectan la toma de decisiones de ellos? Viniendo de Estados Unidos donde periódicamente hay reformas que pretenden legislar la enseñanza, me interesa contribuir a contestar la pregunta de si, dada una reforma, podemos anticipar su éxito o fracaso. O bien, más constructivamente, dada una reforma, me interesa saber cómo podemos identificar qué es lo que hace falta que las organizaciones educativas aprendan sistémicamente para que la reforma tenga éxito (Bryk et al., 2015). ¿Qué conceptos nos permiten anticipar los problemas que va a tener la introducción de una reforma y cómo podemos estudiar la implementación de reformas? Las reformas tienden a partir de lo general e ir a lo particular, empezando con grandes ideas como descubrimiento o equidad, pero con poca claridad sobre los obstáculos y recursos para cambios en lo particular, ¿cómo podemos pensar en esos cambios en lo particular?

Ante la pregunta de si es posible mejorar la enseñanza, el enfoque de la racionalidad de la práctica provee elementos de diseño e interpretación que permiten pensar en el mejoramiento de la enseñanza; partiendo de lo particular para ir a lo general. Los recursos sociotécnicos de diseño de las situaciones de instrucción y de las estructuras institucionales en las que se desempeñan los maestros pueden dar un punto de apoyo para cambios incrementales en la instrucción.

Un ejemplo de recursos institucionales es el currículo designado a través de los libros de texto. El currículo usualmente se ve como un punto de apoyo para las reformas. Pero, hay otras estructuras institucionales, o micro-estructuras en la clase, que pueden servir para la implementación de reformas o más generalmente para mejorar la enseñanza. Nuestro enfoque en la racionalidad de la práctica nos permite desarrollar herramientas teóricas y metodológicas para describir, explicar, y predecir el trabajo del maestro en matemáticas atendiendo la especificidad de su trabajo, la especificidad matemática, situacional, e institucional. 
El estudio de la racionalidad de la práctica es parte de la investigación básica en educación matemática en la medida que busca entender una realidad social que existe independientemente de nuestra voluntad. Más aún, buscamos hacer investigación básica en educación matemática tratando de acercarnos a los estándares de investigación en las ciencias sociales. En particular, avanzando hacia el uso de diseños más rigurosos de investigación y el uso de medición, eventualmente buscando producir explicaciones causales mediante el uso de diseños experimentales.

\section{DESCRIBIENDO LA ESPECIFICIDAD MATEMÁTICA DE LA PRÁCTICA}

El primer problema que tratamos de describir y explicar es ¿qué está haciendo el maestro cuando gestiona la instrucción? Disciplinas como las matemáticas, la pedagogía general, o las ciencias del aprendizaje sugieren describir la práctica como una aplicación de ideas más generales: estas disciplinas dirían que lo que ocurre es el tratamiento de un tópico matemático o la aplicación de alguna pedagogía. El ejemplo dado en las Figuras 1 y 2 podría describirse desde el punto de vista de las matemáticas diciendo que la clase está por probar un teorema de geometría, o que la clase usará la definición de bisectriz para deducir las propiedades de una figura. Desde el punto de vista de la pedagogía general se podría decir que los alumnos han recibido un problema del nivel de análisis en la taxonomía de Bloom o que el maestro está organizando a los estudiantes para un trabajo cooperativo. Nuestro enfoque en la racionalidad de la práctica, por el contrario, desarrolla una teoría de la práctica, buscando describir lo que está en juego para el maestro. ¿Cuál es el problema que el maestro está resolviendo al actuar en la clase? Para el maestro, el problema de la gestión de la enseñanza es más local y más complejo que lo que plantean las perspectivas disciplinares mencionadas.

El problema es local en el sentido de que las decisiones que toma el maestro, por ejemplo la decisión de cómo enunciar el problema planteado en Figuras 1 y 2, es una decisión específica para una clase particular y para conocimientos particulares que el maestro debe de gestionar con los alumnos. La decisión no se reduce a una cuestión de cuál estilo general de enseñanza está por aplicarse (por ejemplo, enseñar por descubrimiento o hacer que los estudiantes trabajen en grupo). 
El problema es más complejo porque las decisiones que toma el maestro requieren una atención a más de una sola cosa. No se trata de una cosa o de la otra: No se trata o bien de trabajar en grupo o bien de probar teoremas, sino de probar un teorema y trabajar en grupo simultáneamente. Esta complejidad sugiere que reducir la investigación en la enseñanza de las matemáticas a las matemáticas, a la pedagogía general, o a las teorías del aprendizaje, implica una desnaturalización del fenómeno.

En vez de esto, considerando la perspectiva de Brousseau (1997, p. 22-23), proponemos describir lo que está en juego para el maestro en términos de la gestión de intercambios entre dos manifestaciones del conocimiento en la práctica de la enseñanza de las matemáticas. Por un lado, hay elementos de conocimiento que los estudiantes tienen que aprender y el maestro debe de enseñar, conocimientos y habilidades que están listados en el currículo. Por otro lado, están las acciones de los estudiantes y sus maestros en tareas particulares, acciones que le dan al maestro evidencia de que los estudiantes tienen (o no tienen) una relación con ese conocimiento. Esas dos maneras de dar cuenta de los conocimientos en juego se manifiestan localmente en la clase y pueden ser usadas para registrar lo que está ocurriendo; pero es posible que, para el caso particular de una lección, ambas formas del conocimiento matemático sean o no sean compatibles. ¿Bajo qué condiciones se puede decir que el concepto que el maestro debe de enseñar a los alumnos está en juego en la tarea que los alumnos están haciendo? ¿Cómo se puede segmentar el trabajo de la clase de tal suerte que éste, consistente en las acciones de alumnos y maestro en una tarea particular, se pueda describir en términos de los objetos curriculares en juego? Para representar este tipo de preguntas hemos propuesto la noción de transacción de instrucción (instructional exchange; Herbst \& Chazan, 2012).

Dado un elemento del conocimiento a ser aprendido, el maestro tiene que crear condiciones para que los estudiantes demuestren que tienen una relación con ese conocimiento. Por ejemplo, el maestro tiene que proponer problemas que al resolverse le den al alumno oportunidad de usar el conocimiento en cuestión. Cuando los estudiantes trabajan en tal problema, el maestro tiene que leer sus acciones en relación al conocimiento en juego. A eso es lo que llamamos un intercambio o una transacción. Es una transacción simbólica entre dos maneras de leer el conocimiento matemático: Como inscrito en la acción y como representado por la acción. 
Brousseau (1997) examina este tipo de intercambios desde el punto de vista del trabajo matemático del estudiante: ¿̇uáles tareas pueden poner a los conocimientos matemáticos en juego y cuándo puede un observador decir que las acciones de los estudiantes representan significativamente los conocimientos matemáticos en juego? Nuestro enfoque intenta resolver el mismo problema desde el punto de vista del maestro. El maestro, desde luego, podría verse como un observador. Sin embargo el maestro es un observador cuya posición institucional lo sesga de manera particular. El maestro no solamente puede estudiar la posibilidad de tal intercambio, sino que al ser responsable del éxito de la enseñanza, necesita que tal intercambio sea posible. Dicho sea de paso, notamos que esta responsabilidad es el producto de una evolución histórica y probablemente también una variable cultural: en Estados Unidos y particularmente después de la ley No Child Left Behind (Bush, 2002), se ha hecho a los maestros responsables de generar evidencia del éxito de la enseñanza. Asumiendo que el maestro debe observar la tarea del estudiante para efectuar tal intercambio, nosotros nos planteamos la siguiente pregunta: ¿̇uáles recursos están disponibles para el maestro y qué restricciones debe de considerar el maestro para gestionar esos intercambios?

\section{LA DECISIÓN DE CÓMO PRESENTAR UN PROBLEMA DE DEMOSTRACIÓN}

Entonces volvamos al ejemplo dado en las Figuras 1 y 2. ¿Qué es lo que está en juego para el maestro? Lo que está en juego para el maestro no es solamente el objeto matemático, la proposición de que las bisectrices de un trapecio isósceles forman un romboide. Esa proposición puede estar en juego para el alumno en una variedad de tareas, pero el trabajo del estudiante también implica una manera particular de conocer la proposición en juego.

En este caso, esta manera de conocer la proposición en juego está anticipada o tal vez sugerida mediante lo que el maestro dice: “¿por qué podríamos decir que las bisectrices de los ángulos de un trapecio isósceles forman un romboide?", o cuando el maestro les pide a los estudiantes que prueben que las bisectrices forman un romboide. En el primer caso, los alumnos podrían pensar en una variedad de justificaciones, mientras que en el segundo caso, la pregunta les requiere proponer una prueba ¿Cuál es la diferencia?

En el segundo caso, poder dar una prueba de la verdad de la proposición, puede darle evidencia al maestro de que el alumno ha adquirido uno de los 
objetos curriculares en juego en la enseñanza de la geometría en Estados Unidos: la habilidad de hacer pruebas. Si usamos la noción de intercambio de instrucción para analizar lo que está pasando en las Figuras 1 y 2 desde el punto de vista del maestro, lo que está en juego es la posibilidad de efectuar una transacción entre dos cosas: por una parte la afirmación de que los estudiantes saben demostrar y por otra parte las acciones específicas que los estudiantes tomen frente a la tarea que involucra las bisectrices de un trapecio isósceles.

Si el maestro puede lograr que los estudiantes demuestren esa proposición, esa evidencia le sirve para decir que los estudiantes saben hacer pruebas. El maestro puede no necesariamente estar interesado en afirmar que los estudiantes conocen la propiedad, pues tal propiedad tiene muy poco valor desde el punto de vista curricular.

Así pues, desde ese punto de vista tal vez se pueda entender un poco mejor por qué la posibilidad dada en la Figura 2b es más probable que la posibilidad dada en la Figura 2a. Mientras que en la posibilidad de la Figura 2a los estudiantes podrían elegir tanto hacer una prueba como proveer una explicación que no fuera una prueba, la posibilidad dada en la Figura 2b requiere una prueba. Esta posibilidad reduce un poco la incertidumbre del maestro de si tendrá evidencia que le permita interpretar el trabajo de los estudiantes.

La misma proposición podría aparecer en otro tipo de intercambios donde los estudiantes no necesitarían probarla. Por ejemplo, el maestro podría decir, "voy a pasar una hoja con un trapecio isósceles. Exploren qué tipo de figura se forma cuando uno traza las bisectrices de los ángulos." El alumno se encontraría con la misma proposición pero de una manera diferente y por consiguiente el tipo de afirmación que el maestro podría hacer, usando las acciones del alumno, sería distinta. En el caso de esta exploración, es improbable que el maestro tenga evidencia de que "los estudiantes saben demostrar." La Figura 3 muestra otra opción, en la que el maestro les pide a los estudiantes que descubran las medidas de los otros ángulos en el diagrama. La Figura 3 representa otra manera de conocer la proposición de que las bisectrices de un trapecio isósceles forman un romboide, en el contexto de lo que llamaríamos situación de cálculo geométrico-numérico (geometric calculation in number; Hsu \& Silver, 2014). El estudiante puede usar las propiedades que conoce sobre ángulos y sobre cuadriláteros, haciendo algunas deducciones para descubrir las medidas de los ángulos. 


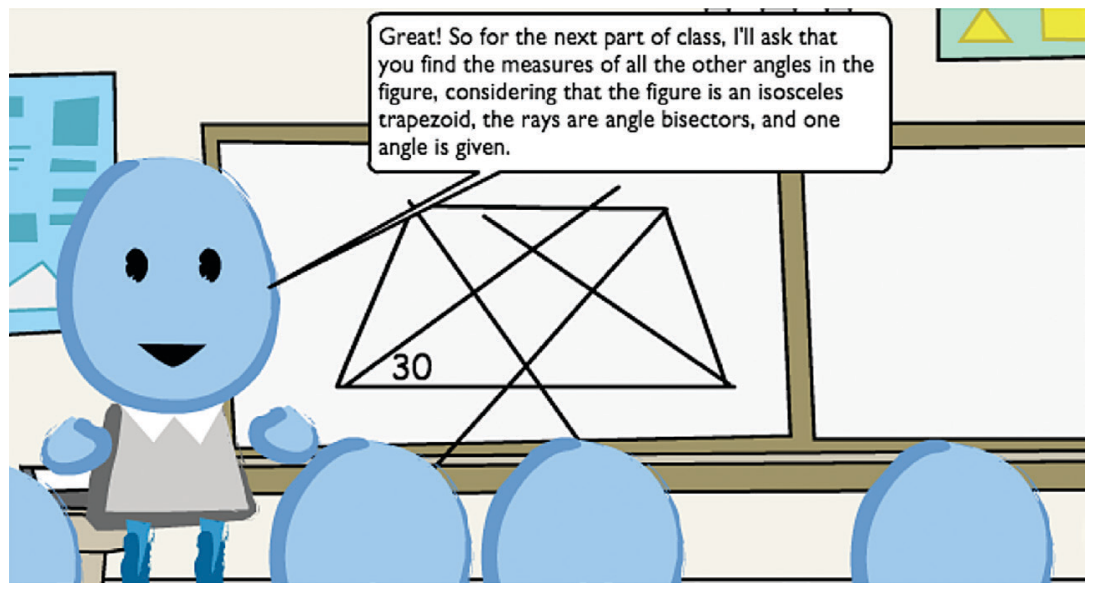

Figura 3. Una tarea de cálculo geométrico-numérico ${ }^{3}$

(C) 2018, The Regents of the University of Michigan, used with permission

Pensando en términos de intercambios entre el trabajo matemático de los estudiantes y las afirmaciones que el maestro puede hacer en relación al currículo, está claro que en cada uno de los casos detallados arriba la misma proposición está involucrada en el intercambio, pero está involucrada de maneras diferentes, pues el trabajo que el alumno va a hacer es probablemente diferente. Por consiguiente, cada una de esas tareas le permite al maestro hacer un intercambio distinto. No estamos hablando de pedagogías alternativas, ni de una elección entre estilos de enseñanza con las cuales se pueda cumplir el mismo objetivo curricular, sino de distintas unidades de conocimiento que son posibles en distintas formas de trabajo, distintas unidades del currículum implementado (Kilpatrick, 1990).

Una teoría de la práctica necesita conceptos que permitan la descripción de éstos intercambios de instrucción en una clase. En la siguiente sección exploro algunos de esos conceptos: marco (framing), contrato, y situación.

3 Traducción: iMuy bien! Durante el resto de la hora, quiero que encuentren las medidas de todos los otros ángulos en la figura, considerando que la figura es un trapecio isósceles, las semirrectas son las bisectrices de los ángulos, y uno de esos ángulos ha sido dado. 


\section{EL MARCO (FRAMING) DE LOS INTERCAMBIOS DE CONOCIMIENTOS: EL CONTRATO}

Un concepto fundamental en el estudio de la racionalidad de la práctica es la noción de framing, que podría traducirse al castellano como marco, y que proviene de la sociología de Erving Goffman. Goffman (1974) observó que el significado de lo que decimos o hacemos depende de las maneras como lo enmarcamos o contextualizamos socialmente; el mismo discurso o las mismas acciones tienen distinto sentido en distintas situaciones. Por ejemplo, la pregunta "¿qué hora es?" tiene un valor transaccional (obtener información acerca de la hora del día) cuando es hecha en la calle a un extraño y otro valor transaccional cuando es hecha en una reunión familiar a un sobrino de seis años (darle al niño la oportunidad de mostrar que él también tiene un reloj o que sabe leer la hora). Nosotros usamos la noción sociológica de marco para buscar maneras de describir el trabajo matemático en la clase.

La noción de contrato didáctico en la teoría de Brousseau (1997) sirve como principio de una explicación al hecho de que las acciones y expresiones en la enseñanza tomen sentido en el marco de la clase de matemáticas. En principio, cualquier persona podría preguntarle a otra ¿qué figura se forma cuando se trazan las bisectrices de los ángulos de un trapecio isósceles? Si tal pregunta se hiciera en el marco de una fiesta donde se está jugando a preguntas y respuestas, es muy probable que la pregunta se juzgue cómica y la respuesta para muchos en la audiencia dé lugar a una multitud de comentarios: "¿Y eso a quien le importa?", "iNo tengo ni la menor idea!", "¿'Una figura geométrica?", "iUna figura horrible!", "iOtra figura!" Pero si la pregunta la hace el maestro en el marco de una clase de geometría, aquélla toma el sentido de una tarea a realizar. Es posible que los alumnos no sepan la respuesta inmediatamente, pero también es razonable que en tal caso se tomen algún tiempo para descubrirla, recordarla, o elegir entre algunas opciones, o, en el peor de los casos, que confiesen no saber la respuesta. El contrato didáctico establece normas muy generales que permiten que estos intercambios ocurran: por ejemplo, el contrato establece que el maestro tiene derecho a (1) asignarles trabajo a los alumnos, (2) observar qué es lo que los alumnos hacen cuando trabajan en la tarea asignada, y (3) emitir un juicio sobre lo que los alumnos hacen. La existencia del contrato didáctico caracteriza a la instrucción: El contrato hace posibles las relaciones entre el maestro, los alumnos, y el conocimiento a enseñar. Al hacerlas posible también les da sentido.

Pero el contrato garantiza las relaciones entre el maestro, los alumnos, y el conocimiento de una manera muy general. En términos de tareas particulares, 
Brousseau observó que una de las responsabilidades fundamentales del maestro es negociar un contrato didáctico local alrededor de cada una de las tareas (o situaciones adidácticas) en las cuales los alumnos van a construir una relación particular con el conocimiento. Una situacion adidáctica pone al alumno en relación con un milieu adidáctico, en el contexto de cuyas interacciones el alumno desarrollará sus conocimientos: esta relación necesitará un contrato didáctico local que le sirva de marco. Tal contrato identificará a cada momento qué se espera del maestro y del alumno, y cómo los elementos del milieu deben de leerse para que den información útil en la formación del concocimiento. Este contrato no es necesariamente estable; el maestro tiene que negociarlo continuamente de tal suerte que se pueda mantener activa la tarea. Es aquí donde mi trabajo comenzó. Me interesaba estudiar las dificultades que tenía que superar el maestro al negociar el contrato didáctico para una tarea.

\section{TENSIONES EN LA GESTIÓN DEL CONTRATO}

Consideremos brevemente el estudio de la tarea "ordenamiento de triángulos" (ranking triangles task; Herbst, 2003): Les dimos a los estudiantes ocho triángulos de cartulina que debían de ordenarse de acuerdo con su área, pero sin usar la fórmula de área o usándola lo menos posible. Inicialmente los estudiantes compararon áreas por inclusión o aditividad. Pero dos triángulos en particular habían sido creados de tal suerte que ninguno de ellos podía ser completamente contenido en el otro. En lugar de eso, un par de lados estaban en una razón 2:1, mientras que las alturas correspondientes a aquellos lados estaban en una razón 1:2; aunque esta información no se les proporcionó a los estudiantes. En el curso del trabajo en la tarea, una estudiante descubrió tales razones y afirmó que los triángulos eran iguales, invocando que al usar la fórmula del área, los factores 2 se cancelarían, y ambas áreas darían el mismo valor. Su compañera, sin embargo insistía que tal razonamiento no era válido pues requería usar la fórmula. Lo que estaba en juego era una nueva relación con la fórmula del área de un triángulono como una manera de producir el valor numérico del área sino como manera de expresar o representar el área de un triángulo particular.

En mi análisis del trabajo del maestro usando esa tarea con los alumnos propuse que la negociación del contrato didáctico para tareas novedosas ${ }^{4}$ como

\footnotetext{
${ }^{4}$ En lo que sigue, tareas novedosas se refiere a tareas novedosas para los estudiantes.
} 
aquélla requiere que el maestro gestione tres tensiones fundamentales. La primera es una tensión entre distintos tipos de objetivos de la tarea-los objetivos comunicados a los estudiantes con la tarea y los objetivos perseguidos por el diseño de la tarea. La segunda es una tensión entre las representaciones de los objetos matemáticos en uso-los objetos concretos de cartulina y los triángulos planos que sirven de modelos matemáticos de aquéllos. La tercera es una tensión entre lo explícitamente desautorizado y lo implícitamente útil-se refiere a las operaciones que los estudiantes pueden pero no deben ejecutar. Estas tres tensiones ilustran un punto fundamental sobre la negociación del contrato didáctico para una tarea novedosa-ésta requiere mucho trabajo de parte del maestro-. Si bien es posible que el maestro negocie el contrato para mantener a la tarea como representación de las matemáticas en juego, también es probable que la tarea se altere como resultado de la necesidad de negociar un contrato didáctico que pueda estabilizar la relación entre el maestro, el alumno, y los conocimientos.

La negociación del contrato didáctico para una tarea novedosa puede requerir mucho trabajo y resultar en alteraciones fundamentales a los conocimientos en juego, lo cual de por sí justifica nuestro interés en estudiar la racionalidad empleada en enseñar clases ordinarias: aquellas clases en las que no se está implementando ninguna intervención sino que el maestro está enseñando tal como lo haría normalmente. Si bien la enseñanza de clases ordinarias también requiere manejar complejidad, la observación de clases ordinarias da cuenta de que hay muchas tareas matemáticas que no parecen requerir tal negociación del contrato. Hay tareas que parecen apelar a contratos locales implícitos que obvian la necesidad de una negociación. Tanto el maestro como los alumnos actúan como si supieran qué deben de hacer. Nos ha interesado caracterizar estos contratos locales pues si ellos sirven para describir períodos de estabilidad de la relación didáctica, sus características pueden ser tremendamente útiles a la hora de predecir y entender las dificultades de gestión del contrato en clases donde se usan tareas novedosas.

\section{SITUACIONES DE INSTRUCCIÓN}

Utilizamos la expresión situación de instrucción (instructional situation) para hablar de aquellos contratos didácticos locales que sirven de marco para el trabajo de los estudiantes en tareas familiares. Una situación de instrucción 
incluye normas específicas al conocimiento, que son, sin embargo, repetibles en el contexto de tareas similares. Tres ejemplos de situaciones de instrucción en la clase de geometría de la escuela secundaria en Estados Unidos son las situaciones de cálculo, de exploración, y de demostración. En cada una de ellas hay ciertas cosas que se mantienen tácitas, es decir que no son objeto de negociación explícita, y que tanto maestros como alumnos actúan como si supieran qué es lo que ellos deben de hacer.

Por ejemplo en la situación de cálculo geométrico-numérico (geometric calculation in number; Hsu \& Silver, 2014) o geométrico-algebraico (geometric calculation in algebra, Boileau \& Herbst, 2015) el maestro les da a los alumnos un diagrama con información acerca de la figura representada, incluyendo las longitudes de algunos lados o las medidas de algunos ángulos, y les pregunta a los estudiantes algo sobre la figura (e.g., cuál es la medida de un lado o de un ángulo). Los estudiantes saben que no se espera que midan nada sino que usen la información dada para calcular sin medir. Por otra parte el maestro sabe que, de alguna manera, tiene que dar información suficiente y no contradictoria para que los alumnos encuentren la respuesta a la pregunta mediante un cálculo. Las situaciones de exploración son muy distintas: En ellas el maestro también da un diagrama o artefacto concreto, pero los estudiantes saben que pueden hacer algo más concreto con la figura, por ejemplo utilizar instrumentos de medición. En situaciones de demostración también se da un diagrama con información distinta de la provista en situaciones de cálculo o de exploración (ver Figura 2b) y se espera que los estudiantes interactúen con el diagrama sin medir.

Cuando hablo de situaciones de instrucción me refiero a los marcos posibles para las tareas matemáticas. Distintas situaciones de instrucción pueden usarse para organizar el trabajo de la clase y cada una de ellas puede enmarcar tareas distintas. Por ejemplo, una situación de exploración puede enmarcar simultáneamente el descubrimiento de propiedades de los ángulos formados por rectas paralelas cortadas por una transversal y el descubrimiento de las propiedades del romboide. Pero lo que nos interesa no es solamente que hay tareas distintas que pueden enmarcarse en la misma situación, sino que esas tareas comparten ciertas regularidades; recurren varias veces durante el año lectivo o año tras año en la práctica docente. A estas regularidades las llamamos normas de instrucción, o más específicamente normas de una situación de instrucción.

Volviendo a la noción del intercambio, una situación de instrucción enmarca el trabajo del maestro de tal manera que éste pueda gestionar el intercambio entre el trabajo que observa hacer a los estudiantes y los objetos de 
conocimiento que están en juego en ese trabajo. El enmarcado de una tarea, en una situación, permite que haya ciertas normas que faciliten la atestación del intercambio, por ejemplo diciéndole al maestro qué cosas ver y qué cosas ignorar, y diciéndole al estudiante qué cosas puede hacer y qué cosas no debe de hacer. En la sección siguiente examinamos un ejemplo.

\section{EL REGISTRO DIAGRAMÁTICO Y LA INVESTIGACIÓN SOBRE LAS NORMAS}

Habiendo introducido la noción fundamental de norma de una situación, ahora podemos volver al problema original y abordar cuestiones metodológicas. La pregunta original era cuál es la manera más probable en la que el maestro enunciará el problema sugerido en la Figura 1, si se espera que los estudiantes hagan una demostración.

\section{El registro diagramático}

A partir de nuestras observaciones de situaciones de demostración en la clase de geometría en Estados Unidos (Herbst, Chen, Weiss, \& González, 2009), hemos conjeturado que la norma de enunciación de problemas de demostración es que éstos se enuncien en lo que llamamos el registro diagramático (diagramatic register; Herbst, Kosko, \& Dimmel, 2013; ver también Boileau, Dimmel, \& Herbst, 2016). Esta norma tiene cinco incisos o subnormas, a saber:

- Que el enunciado del problema no haga referencia explícita a propiedades de incidencia, orden, separación, colinearidad, o concurrencia, las cuales se dejan libradas al diagrama para comunicar (véase Manders, 1995).

- Que el maestro provea un diagrama para que los estudiantes lo usen cuando hagan la prueba en vez de dejar que los estudiantes hagan un diagrama.

- Que el diagrama provisto por el maestro sea tal que todos los puntos que se necesitarán usar para enunciar las propiedades dadas o a probar y para hacer la prueba hayan sido identificados con letras, aunque no todos los puntos del diagrama sean identificados con letras.

- Que tanto la proposición dada como hipótesis (datos) como la proposición a probar se enuncien utilizando símbolos y letras que identifican a objetos 
en el diagrama, en lugar de palabras que representan a los conceptos involucrados.

- Que el diagrama sea suficientemente exacto para que las proposiciones dadas y a probar puedan ser creíbles al ver el diagrama.

Estos incisos son parte de una conjetura sobre la norma de instrucción: Conjeturamos que la norma es que los problemas de demostración se enuncien siguiendo tal norma. Es una conjetura descriptiva, pues intenta describir una realidad. Más aún, es una conjetura ya que está supeditada a su verificación empírica. En lo que sigue describo cómo emprendemos la verificación empírica.

Consideremos la decisión entre las dos posibilidades dadas en la Figura 2. Si comparamos nuevamente los enunciados de las Figuras 2 a y $2 b$, hay dos cosas en particular en el enunciado dado en $2 a$ que lo hacen menos probable que el enunciado de la Figura 2b: primero, el diagrama no incluye un diagrama del romboide y segundo la proposición está enunciada conceptualmente, transgrediendo así los incisos segundo y cuarto enunciados más arriba. En el problema presentado en la Figura $2 b$ se incluye el diagrama del romboide y letras identificando los vértices del trapecio y del romboide. Sin embargo, el enunciado de la derecha también expresa la propiedad a probar en términos conceptuales, no en términos de los objetos en el diagrama. Así pues, el enunciado de la derecha tampoco se adhiere totalmente a la norma del registro diagramático, aunque está más cercano a esa norma. Sin embargo, el hecho de que el enunciado además usa el imperativo "prove" (demuestre) para indicar qué se espera del estudiante (en contraste con el enunciado de la izquierda que sólo pregunta "por qué?"), hace que podamos predecir que quienes han sido socializados en la norma (por ejemplo, maestros que tienen experiencia enseñando geometría en Estados Unidos), reconocerán la opción de la derecha (Figura 2b) como la más apropiada (ver Herbst et al., 2009).

Las alternativas mostradas en la Figura 2 son dos de las cuatro alternativas que les ofrecemos a los maestros que responden a nuestros cuestionarios de toma de decisiones. Ellos ilustran cómo funciona la noción de norma en el diseño de preguntas en un cuestionario para investigar la toma de decisiones. Si se plantea tomar una decisión en un momento en el que la decisión se supone afectada por una norma, las opciones de cumplir con la norma o transgredir la norma se pueden usar para generar las alternativas de la decisión.

Ante las nociones de situación y de norma, uno se podría preguntar ¿para qué nos sirven? Las nociones de transacción o intercambio, contrato, y situación 
nos permiten describir la matemática que está en juego para el maestro en la toma de decisiones. La noción de norma nos permite describir las demandas específicas de la situación en la que se toma la decisión. Podemos pensar la instrucción matemática en un curso de estudios como una sucesión finita de transacciones, muchas de las cuales se facilitan mediante situaciones de instrucción que se suceden durante el año, mientras que unas pocas de ellas se facilitan mediante tareas que son novedosas y para las cuales es menester negociar el contrato didáctico.

Entonces si queremos entender el costo de una decisión, podemos considerar ¿qué tipo de transacción?, ¿en cuál contrato?, ¿en cuál situación?, ¿̇en qué tipo de transacción se ubica la decisión que se va a tomar? Y si la transacción se enmarca dentro de una situación de instrucción, ¿en qué medida la decisión a tomar transgrede una norma de la situación?

Esa es una manera de operacionalizar el problema de toma de decisiones dentro de la teoría. En el ejemplo de las bisectrices de los ángulos del trapecio isósceles, ambas decisiones se desvían de la norma, pero de manera distinta. Y se podría decir que la decisión mostrada en la Figura 2a se desvía mucho más que la de la Figura 2b. Por supuesto que eso no quiere decir que todo el mundo tomará la misma decisión. Nos interesa dar cuenta del fenómeno de toma de decisiones, y para ello la noción de norma es útil: nos permite predecir decisiones posibles mediante anticipar distintas maneras como la norma podría dejar de cumplirse.

El maestro puede, por supuesto, decidir hacer algo que implica una desviación de la norma, en cuyo caso predecimos que le costará más trabajo lograr que el estudiante haga lo que el maestro espera. Pero si el maestro presentara el enunciado de la izquierda (Figura 2a), puede ser que haya cosas que ganar también: imagínese que un maestro les pregunte a los estudiantes por qué se puede decir aquello y ellos le expliquen que la simetría del trapezoide isósceles hace que sus bisectrices generen un cuadrilátero con dos pares de lados congruentes. Puede que la tarea del maestro se haga más difícil, pero también puede abrir un espacio para generar evidencia de que los estudiantes están pensando racionalmente sobre la geometría.

Pero concentrémonos en la norma por un momento. La pregunta empírica es ¿cómo sabemos que tales normas lo son? Hasta ahora he afirmado que aquéllas son las normas, pero también he dicho que la noción de norma no es prescriptiva, sino descriptiva. ¿Cómo verificamos tal hipótesis descriptiva? 


\section{EXPERIMENTOS VIRTUALES DE TRANSGRESIÓN EN LÍNEA}

Una de las herramientas metodológicas que hemos adaptado es la noción de breaching experiment, o experimento de transgresión, concebida originalmente por Harold Garfinkel (ver Mehan \& Wood, 1975) para estudiar cómo la gente negocia transgresiones en las normas sociales (por ejemplo en relación a cómo obtener una mesa en un restaurante). Garfinkel llama repair strategies (o, en castellano, reparos u objeciones) a las acciones que sugieren la necesidad de negociar el hecho de que una norma no se ha cumplido. La existencia de tales reparos sirve de evidencia para observar que una norma se ha transgredido, lo que por su parte contribuye a la evidencia de que tal norma existe.

Nosotros hemos adoptado la metodología de los experimentos de transgresión para hacer investigación sobre las normas de la instrucción en matemáticas. Comenzamos haciendo este trabajo en clases de geometría, utilizando intervenciones donde, por ejemplo, uno de nosotros ocupaba el rol de maestro y gestionaba la instrucción de manera tal que alguna de las normas de una situación no se cumplieran. Por ejemplo, en la lección analizada por Herbst \& Chazan (2003) tomé yo mismo el papel del maestro y alenté a un estudiante a que prosiguiera escribiendo las afirmaciones de una demostración a pesar de que el estudiante no había podido justificar la última afirmación enunciada. La norma hipotética es que en la escritura de una demostración, cada enunciado debe de ser acompañado de su justificación antes de que se emita el siguiente enunciado (véase Herbst, et al., 2009). Este experimento de transgresión tenía como objetivo, entre otras cosas, ver cómo se adaptaba el resto de la clase, incluyendo el maestro titular que estaba presente, a una situación de demostración que se desviara de la norma, cómo el maestro daba cuenta de que había existido una desviación, y cómo reparaba la presunta transgresión. Otros experimentos en clase incluyeron colaborar con maestros en diseñar e implementar lecciones y hasta unidades de estudio donde los problemas enunciados contradecían las normas de instrucción. Por ejemplo, en Herbst $(2005,2006)$ se describen unas lecciones sobre área en las que la maestra les pidió a los estudiantes que probaran un resultado en un dominio de conocimientos (área de figuras geométricas) para el cual los estudiantes no tenían acceso a postulados o definiciones que pudieran usar como justificaciones de los enunciados que ellos deberían de incluir en la demostración. En estos casos, nuestro objetivo también era ver como la clase y la maestra se adaptaban a trabajar 
en un contexto donde las normas usuales no fueran cumplidas, lo que de paso nos permitió confirmar nuestras conjeturas de que aquellas normas estaban presentes: en este caso que la norma que los enunciados en una demostración deben de justificarse usando definiciones, postulados, y teoremas introducidos con anterioridad.

Luego de hacer algunos experimentos de transgresión en clases, hemos buscado maneras de obtener mejor acceso a las perspectivas con las que los maestros responden a las transgresiones de una norma. Para ello hemos desarrollado la técnica de experimentos virtuales de transgresión, utilizando videos de situaciones en clase donde una norma se había transgredido y presentándoselas a maestros reunidos en grupos focales. Nuestro objetivo ha sido ver cómo los maestros describen lo que ven, cómo discuten entre ellos qué es lo que estaba pasando. Al gestionar esos grupos focales nosotros no decimos nada que sugiera que pensamos que una norma se ha transgredido, pero grabamos y analizamos el discurso de los maestros para confirmar que las normas existen (Nachlieli \& Herbst, 2009; Weiss, Herbst, \& Chen, 2009).

En una tercera iteración de nuestro uso de experimentos de transgresión, comenzamos a representar instancias de transgresión usando dibujos animados, con los caracteres mostrados en las Figuras 1 y 2, en lugar de videos. Continuamos presentando estos videos a grupos focales, pero la decisión de representar acciones usando caracteres animados nos ha permitido ser más sistemáticos en nuestra exploración de conjeturas. Los artículos de Herbst, Nachlieli, y Chazan (2011) y de Chazan y Herbst (2012) muestran como hemos analizado las respuestas de los grupos focales, para entender la racionalidad de la práctica. El artículo de Herbst y Kosko (2014b) muestra que las respuestas de los maestros a las animaciones son comparables a las respuestas que ellos dan a videos en lo que concierne a sus reparos sobre las transgresiones de las normas. Sin embargo, tanto estos experimentos virtuales con animaciones como los anteriores con videos solamente sirven como prueba de la existencia de un fenómeno. El paso siguiente ha sido definir constructos y medidas relacionadas con este fenómeno, para lo cual hemos buscado una manera de recabar datos en mayor cantidad, usando lo que llamamos experimento de transgresión virtual en línea (online virtual breaching experiment; Herbst \& Chazan, 2015).

En nuestros estudios más recientes, hemos estado utilizando los mismos caracteres animados que antes usábamos en las animaciones para crear tiras cómicas que muestran una situación de instrucción en la que una norma se ha transgredido y tiras cómicas en las que ninguna norma se ha transgredido, 
como control. Distribuimos esas representaciones en línea ${ }^{5}$ les pedimos a los participantes que describan lo que ven. Examinamos lo que los maestros contestan en respuesta a estas representaciones, buscando evidencia de que reconocen que una norma de instrucción ha sido transgredida. Dimmel y Herbst $(2017,2018)$ utilizan esta técnica en el contexto de un diseño experimental, es decir con asignación aleatoria de participantes a dos condiciones distintas: ver representaciones de una situacion con transgresiones o verlas sin transgresiones. Del análisis de las respuestas se obtienen medidas de reconocimiento de la norma que luego pueden ser analizadas usando métodos estadísticos (p. ej., t-tests).

\section{EVALUANDO LA NORMA DEL REGISTRO DIAGRAMÁTICO}

En lo que sigue, me concentro en el registro diagramático descrito anteriormente. Para estimar en qué medida alguien reconoce la norma del registro diagramático, hemos creado ítems que incluyen una tira cómica en la que se representa una situación de demostración y donde la norma del registro diagramático ha sido transgredida. Le pedimos al participante que primero describa lo que ha visto y después que evalúe cuán apropiada ha sido la acción del maestro (usando una escala numérica). Codificamos las descripciones buscando evidencia lingüística de que el maestro reconoce la transgresión, mediante, por ejemplo, mencionarla explícitamente, diciendo qué fue lo que el maestro no hizo y qué debería de haber hecho. Con esas codificaciones construimos medidas. Una medida es el reconocimiento de la norma (norm recognition score), que es la cantidad (o el promedio) de los ítems en los que el maestro reconoce la transgresión de la norma. Otra medida es cuán apropiada es la acción (appropriateness score) que es el promedio de las evaluaciones que los participantes ofrecen a cada una de las representaciones mostradas.

Esas definiciones se vuelven operativas en versiones de un instrumento al que llamamos Reconocimiento Implícito de la Norma o INR por sus siglas en inglés (Implicit Norm Recognition). En cada una de las versiones de INR ${ }^{6}$ ofre-

\footnotetext{
${ }_{5}$ La plataforma LessonSketch (www.lessonsketch.org) nos ha permitido crear las tiras cómicas y los cuestionarios, asimismo como distribuir los cuestionarios a nuestros participantes y obtener reportes con sus respuestas.

${ }_{6}$ Distintas versiones de INR miden el reconocimiento de normas diferentes. Para la situación de demostración hemos generado cuatro versiones.
} 
cemos cuatro representaciones de la situación de demostración en la forma de tiras cómicas que muestran cómo el maestro organiza una situación de demostración, y en particular cómo enuncia el problema. Para cada una de ellas les pedimos a los participantes que (1) describan lo que ven en la tira y (2) que evalúen cuán apropiada creen ellos que es la acción del maestro, usando una escala de 1 a 6 . Entre las versiones del INR que hemos desarrollado está el INR (DP-DR) que consiste en cuatro tiras cómicas que muestran instancias en las que la norma del registro diagramático ha sido transgredida, y el INR (DP-C) en el cuál las cuatro tiras muestran instancias en que la situación de demostración se ejecuta sin transgredir ninguna norma. Para cada uno de ellos es posible calcular medidas promedio de reconocimiento de la norma y de lo apropiado de la acción del maestro, las cuales se pueden comparar utilizando pruebas de hipótesis para la diferencia de las medias en cada medida, donde la hipótesis nula es que no hay diferencia entre los dos grupos (diferencia de medias es 0). En Herbst, Dimmel, y Erickson (2016) reportamos que hay evidencia de que las medidas de reconocimiento de la norma son significativamente mayores cuando los maestros responden a representaciones de transgresión (el caso del instrumento INR (DP-DR)) que cuando responden a representaciones donde la norma se ha mantenido (el caso del instrumento INR (DP-C)). Esto sugiere que es más probable que los maestros reconozcan la norma cuando presencian su transgresión, que cuando presencian su cumplimiento. Además, Herbst, Dimmel, y Erickson (2016) encontraron diferencias en las medidas de lo apropiado de la acción: Ios participantes consideraron significativamente más apropiada las acciones del maestro cuando cumplió con la norma que cuando no la cumplió.

Recientemente hemos utilizado los cuestionarios en línea basados en tiras cómicas para estudiar una variedad de fenómenos en álgebra y en geometría: normas relativas a la presentación de los enunciados de prueba en geometría, la importancia del método de resolución de ecuaciones en álgebra, o la importancia del tipo de registro en la resolución de problemas de palabras en álgebra. También hemos utilizado las tiras cómicas para plantear problemas de toma de decisiones, donde, por ejemplo, se representa una situación hasta el momento en que una norma se debería de aplicar, y luego se le pide al participante que elija una de entre cuatro posibles maneras de actuar -una de ellas es la normativa mientras que las otras son otras acciones posibles-. Los artículos de Herbst, Chazan, Kosko, Dimmel, y Erickson (2016) y Erickson y Herbst (2016) muestran resultados preliminares del análisis de esos cuestionarios. Finalmente, 
también hemos utilizado tiras cómicas para obtener datos que informan en qué medida los maestros están dispuestos a justificar acciones que se distancian de una norma para atender a una obligación profesional.

\section{DESVIACIONES DE LA NORMA Y SUS JUSTIFICACIONES}

La afirmación hecha hasta aquí es que las tareas matemáticas que se enmarcan dentro de situaciones de instrucción requieren menos negociación del contrato didáctico que las tareas novedosas. Las situaciones de exploración y de demostración son ejemplos de esas situaciones de instrucción. Esas situaciones contienen expectativas para las decisiones del maestro o normas que se aceptan tácitamente, es decir, si las acciones cumplen con la norma la gente tiende a no mencionar que una norma se ejecutó, pero si las acciones no la cumplen, tales acciones atraen atención a sí mismas, generando la necesidad de negociar el contrato. La norma "en situaciones de demostración el problema se enuncia utilizando el registro diagramático" es un ejemplo de esas expectativas. Y como mostramos más arriba, hay maneras de verificar la existencia de la norma empíricamente. Las situaciones de instrucción pueden describirse como segmentos de prácticas especializadas en ese sentido: segmentos de prácticas caracterizadas por un conjunto de normas que regulan la actividad matemática y que se aceptan tácitamente.

Ahora bien, obviamente los maestros son personas con libre albedrío. El hecho de que aquellas situaciones de instrucción con sus normas existan no quiere decir que sea obligatorio para un maestro cumplir con esas normas. Al actuar en la instrucción, tanto los maestros como los alumnos podrían desviarse de la norma, pues esas normas no son leyes físicas que actúen contra la voluntad, ni leyes legales cuya transgresión active la acción represiva del Estado. Son normas sociales (o mejor, sociotécnicas) que sugieren un camino que parece como el más natural o sencillo desde el punto de vista de los maestros; su transgresión puede tener un costo (por ejemplo, el ridículo o la frustración) pero tal vez también un beneficio (por ejemplo, la novedad, la diversión, o como vemos más adelante, la satisfacción de una obligación profesional). La existencia de esas normas se debe a probablemente razones históricas y culturales. Esto sugiere, por lo tanto, que los maestros pueden transgredir una norma dentro de una situación, e incluso, que pueden transgredir tantas normas que la situación no proporcionará ya ninguna ayuda para evitar la negociación del contrato. 


\section{DESVIACIÓN DE LAS NORMAS Y PROMOCIÓN DEL APRENDIZAJE}

Desde el punto de vista de la promoción del aprendizaje, ese tipo de transgresiones de las normas son necesarias: si las situaciones donde todas las normas se cumplen les dan la oportunidad a los estudiantes para que practiquen lo que saben hacer, tiene que haber otras oportunidades en las que los estudiantes puedan aprender a hacer cosas que no saben. Una consecuencia teórica de esto es la predicción de que al menos en algunos momentos discretos durante el año, las situaciones de instrucción que han estado sirviendo para organizar el trabajo que los estudiantes saben hacer se tendrán que transgredir de alguna manera. Esto sugiere que hay una especie de dialéctica entre la práctica y el aprendizaje en tanto cambio progresivo de prácticas. En esta dialéctica, el uso de situaciones y sus normas se alterna con transgresiones de algunas normas y negociación del contrato para establecer nuevas situaciones.

Las nociones de contrato, situación, y norma, tal como han sido usadas hasta ahora podrían parecer un poco estáticas en la medida que describen prácticas estables. La noción de que la promoción del aprendizaje por parte del maestro se puede operar en términos de la transgresión de normas en situaciones y contratos, permite asimilar la promoción de los aprendizajes a una teoría de la práctica. Aquellas normas importan no tanto como requisitos sino como recursos para la acción del maestro; son recursos que la actividad de instrucción hace disponible al maestro para decidir qué hacer y qué dejar de hacer. Más aún, en la medida que el trabajo en organizaciones como los salones de clase es dinámico, la transgresión de las normas puede ser un hecho relativamente frecuente.

¿Cómo podemos utilizar las nociones de norma, situación, y contrato para describir una realidad más dinámica, como la toma de decisiones en instrucción? Volviendo al ejemplo descrito en las Figuras 1 y 2, supongamos que el maestro les diga a los estudiantes que copien el diagrama del tablero y trabajen con el compañero en el problema dado en el caso mostrado en la Figura 2a. Tal decisión podría ser deseable ya que podría ser beneficiosa para el aprendizaje, aunque tal beneficio no está asegurado. Además, en la medida en que tome tal decisión el maestro transgredirá una o más normas, podemos predecir que esa elección tendrá ciertos costos. Dado que la decisión es viable, que un maestro la puede tomar y generar con ella cierta actividad de parte de los estudiantes, cabe preguntarse cómo podría justificarse esa decisión: ¿Bajo qué 
consideraciones sería razonable para un maestro presentar el problema así? Podemos pensar esta pregunta como una cuestión de recursos: ¿Qué tipo de recursos tiene el maestro a su disposición para justificar el costo de transgredir una norma?

\section{RECURSOS PARA JUSTIFICAR LAS TRANSGRESIONES DE LA NORMA: OBLIGACIONES PROFESIONALES}

El conocimiento y las creencias del maestro son recursos personales que le permiten componer acciones que pueden desviarse de la norma. El diagrama en la Figura 2a, por ejemplo, no es para nada común y alguien tiene que tener la idea de dibujar el diagrama de esa manera. El origen de la transgresión puede estar en tales recursos individuales, así como en recursos institucionales como las notas de clase de un colega o un libro de texto alternativo. Pero la transgresión de la norma requiere trabajo extra, incluyendo la posibilidad de tener que renegociar el contrato didáctico para la tarea. ¿Qué recursos le permiten al maestro justificar tal decisión, considerando que el costo de la transgresión es mayor que el costo de cumplir con la norma? ¿Y a qué nos referimos con mayor costo? Puede ser que se necesite más tiempo para que los estudiantes resuelvan un problema que no se atiene a la norma, y la necesidad de más tiempo puede impedir la coordinación del paso al que van en las lecciones con colegas que enseñan el mismo curso a otros grupos de estudiantes. Pero ante tal costo, por ejemplo ante el costo institucional en tiempo y la posible pérdida de coordinación con otras secciones del mismo curso, seguramente hay otras consideraciones que sugieren un beneficio. ¿Qué beneficio podría tener el presentar un problema en la manera del caso de la Figura 2a? Una posibilidad es el aliciente de que los estudiantes tendrían la oportunidad de pensar en las razones antes de visualizar la figura, o que tendrían que usar razones para corregir la visualización de la figura que obtengan al extender las bisectrices. Éstos son alicientes matemáticos-el diagrama en la Figura 2a parece que daría más oportunidad a los alumnos de tener una experiencia con la demostración en tanto proceso de descubrimiento razonado.

Herbst y Chazan (2012; véase tambien Chazan, Herbst, y Clark, 2016) han propuesto la noción de obligaciones profesionales (professional obligations) para identificar recursos para la justificación de las acciones con las que podemos 
formalizar estas nociones de costo y beneficio. De la sociología de Bourdieu (1990) tomamos la noción de disposición, para describir tendencias que son vivenciadas como naturales por individuos, pero que se relacionan con la posición social que aquéllos ocupan. En el caso de los maestros de matemáticas, por ejemplo, hay quienes están dispuestos a utilizar solamente el libro de texto como fuente de problemas y hay quienes buscan problemas fuera del libro. Ambas opciones son, por supuesto, concebibles y aceptables, pero lo que nos interesa es que ellas subtienden un espacio de posibilidades en el cuál se espera que los maestros tengan una disposición, aun si las disposiciones particulares son distintas entre colega y colega: se espera que los maestros tengan una disposición hacia el uso del libro como fuente de problemas.

Hay muchos asuntos en los que legítimamente se puede esperar que los maestros tengan una disposición: Aquellos que atañen a su actividad profesional. Esos espacios en los cuales podemos esperar que los maestros de matemáticas tengan una disposición los agregamos en cuatro conjuntos a los que llamamos obligaciones profesionales. Uno de esos espacios es la matemática como disciplina (disciplinary obligation) -los maestros de matemáticas tienen una obligación con la disciplina matemática: por ejemplo, es razonable esperar que los maestros tengan una disposición acerca de la importancia de la precisión en el uso del lenguaje matemático. Un segundo espacio es el de los estudiantes como individuos (individual obligation)- los maestros tienen una obligación con los estudiantes en tanto personas individuales: por ejemplo, es razonable esperar que los maestros tengan una disposición acerca de cómo atender a las necesidades emocionales de los estudiantes. Un tercer espacio es el de la clase como grupo social (interpersonal obligation)-los maestros tienen una obligación con la clase como colectivo interpersonal: por ejemplo, es razonable esperar que los maestros tengan una disposición acerca de la calidad del lenguaje que los estudiantes usan al hablar, entre ellos, en la clase. Y finalmente el espacio de las instituciones educativas (institutional obligation)--los maestros tienen una obligación con las instituciones que organizan la educación, sean ellas departamentos, escuelas, distritos, o sistemas educativos: por ejemplo, es razonable esperar que los maestros tengan una disposición acerca de los requerimientos del calendario académico.

Nuestra afirmación es que esas cuatro obligaciones profesionales reúnen aspectos de la práctica profesional para los cuales los maestros deben de tener una disposición: La posición profesional de ellos los obliga (si bien estas obligaciones no están escritas en contratos) a estar dispuestos a responder a 
cuestiones que emanan de aquellos cuatro espacios (si bien la disposición particular de un maestro puede ser distinta de la de otro). Afirmamos que esas obligaciones agrupan disposiciones que proveen recursos para la justificación de la acción. Una manera de utilizar esta noción de obligación profesional, entonces, es decir que cuando el maestro toma una decisión que transgrede una norma, tal decisión se puede justificar o criticar en referencia a alguna de estas obligaciones-por ejemplo, que la decisión tiene un costo o que provee un beneficio relativo a alguna de esas obligaciones.

El problema en la Figura 2a podría preferirse antes que el problema en la Figura 2b atendiendo a la obligación con la disciplina, pues da la oportunidad de observar a los estudiantes haciendo un trabajo matemáticamente más interesante. El problema de la Figura 2a también requiere a los estudiantes que experimenten la necesidad de convenciones para hacer posible la comunicación: dado que no hay letras en el diagrama, si los alumnos no ponen las mismas letras, les será muy difícil poder trabajar juntos. Si el maestro prefiriera usar ese problema, podríamos entender esta decisión como una desviación de la norma que también es justificable atendiendo a una obligación a la clase como un grupo social, por cuanto les da a los alumnos una oportunidad para aprender a comunicarse.

Para cada una de las cuatro obligaciones profesionales, nosotros definimos el constructo reconocimiento de una obligación profesional y lo medimos utilizando cuestionarios que tienen entre 15 y 18 ítems cada uno. Cada ítem incluye una escena de instrucción dada con una tira cómica, en la cual el maestro ha hecho algo que se desvía de la norma, y una afirmación del tipo "el maestro debería de haber [hecho lo normativo] en lugar de [hacer lo que hizo]." Se les pide a los participantes que indiquen su grado de acuerdo con la afirmación en una escala de 1 a 6 y que comenten su evaluación usando una respuesta abierta. Los valores que ellos le dan a cada ítem se utilizan para proveer una estimación del grado en el cuál reconocen la obligación en cuestión, o sea en la medida en que son capaces de justificar a los maestros por no haber seguido la norma, y tomamos el promedio de esos puntajes como una medida del reconocimiento de una obligación particular (Herbst et al., 2014; Herbst, Chazan, Kosko, Dimmel, \& Erickson, 2016). 

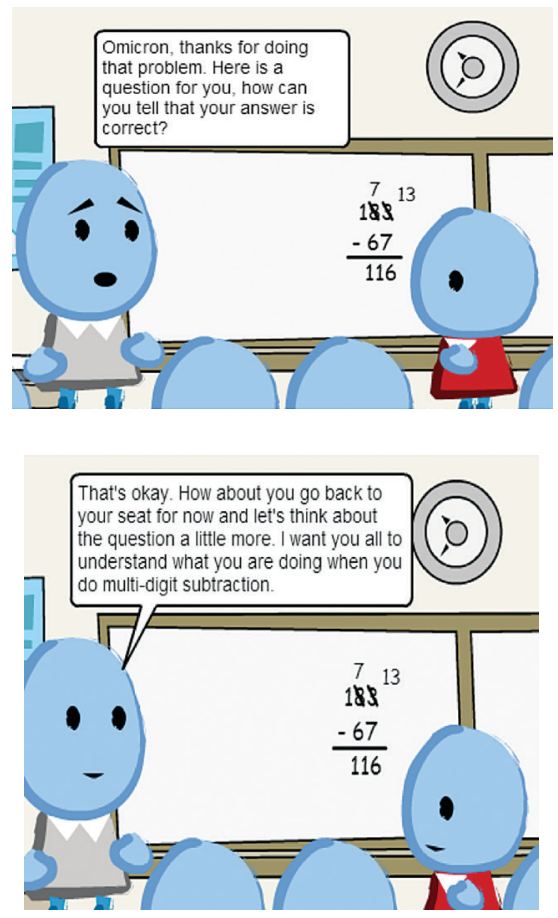

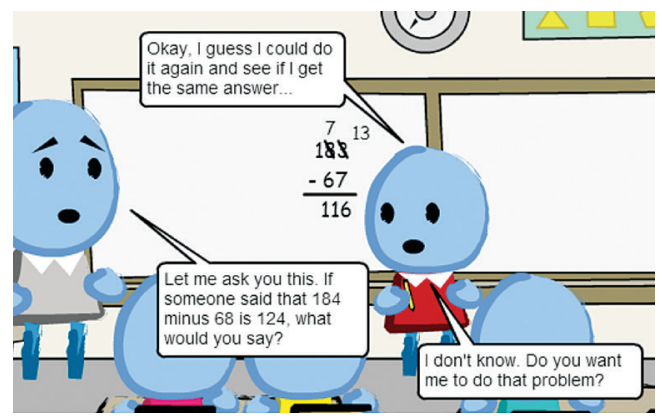

Indique su grado de acuerdo con la siguiente afirmación:

El maestro debía de haber aceptado la solución correcta del estudiante en lugar de hacerle una pregunta distinta

1 -total desacuerdo ...

6-total acuerdo

Comentario:

Figura 4. Un ítem de reconocimiento de obligaciones profesionales ${ }^{7}$

C 2018, The Regents of the University of Michigan, used with permission

Por ejemplo, en la Figura 4 tenemos una de estas escenas presentada en el contexto de una clase de primaria. El maestro aquí ha transgredido una norma: si bien el estudiante resolvió el problema correctamente, el maestro no le ha dicho nada sobre la calidad del trabajo que hizo el alumno. Si lo que hizo el

\section{Traducción:}

Cuadro 1: Maestro: Omicron, gracias por hacer ese problema. Tengo una pregunta para ti... ¿̇cómo podrías saber si tu respuesta es correcta?

Cuadro 2: Omicron: Me imagino que podría hacer de nuevo el problema y ver si da lo mismo. Maestro: Y si alguien te dice que 184 menos 68 es 124, ¿̇ué le dirías? Omicron: No sé. ¿Quiere usted que haga ese problema?

Cuadro 3: Maestro: Está bien. ¿Qué tal si te sientas por ahora y piensas en la pregunta un poco más? Me interesa que todos ustedes entiendan lo que están haciendo cuando resuelven problemas de sustracción con varias cifras. 
maestro fuera justificable, ¿cómo se justificaría? Nosotros les pedimos a nuestros participantes que digan en qué medida están de acuerdo con la afirmación que el maestro debería de haber aceptado la solución correcta del estudiante en lugar de darle una pregunta distinta.

Si los maestros que responden al item en la Figura 4 reconocieran una obligación a la disciplina de las matemáticas sería más probable que estén muy en desacuerdo con la afirmación en la esquina inferior derecha de la Figura 4: En la medida que las matemáticas deberían permitir al alumno usar lo que hizo en el tablero para dar una opinión sobre el segundo problema presentado, la segunda substracción tiene que dar el mismo resultado y si el estudiante lo ve como un problema totalmente distinto, se ha perdido algo a pesar de que haya podido ejecutar la primera operación aceptablemente. Utilizamos una serie de ítems como éste y obtenemos así medidas que dan cuenta de cuán capaces son los maestros de usar una obligación dada para justificar acciones que transgreden normas. Tenemos instrumentos para estimar el reconocimiento de cada una de las obligaciones para cinco niveles de escolaridad, elemental baja, elemental alta, escuela media, escuela secundaria (high school), y universidad.

\section{LA TOMA DE DECISIONES}

El estudio de la toma de decisiones en una situación de instrucción, requiere la hipótesis de una norma de instrucción que indica la acción esperada y de alternativas a tal acción que se desvían de la norma. Operamos estas alternativas utilizando la noción de obligaciones profesionales. Alternativas razonables a la norma pueden encontrarse mediante la hipótesis de que la transgresión de una norma puede tener como beneficio atender a una obligación profesional en particular. 

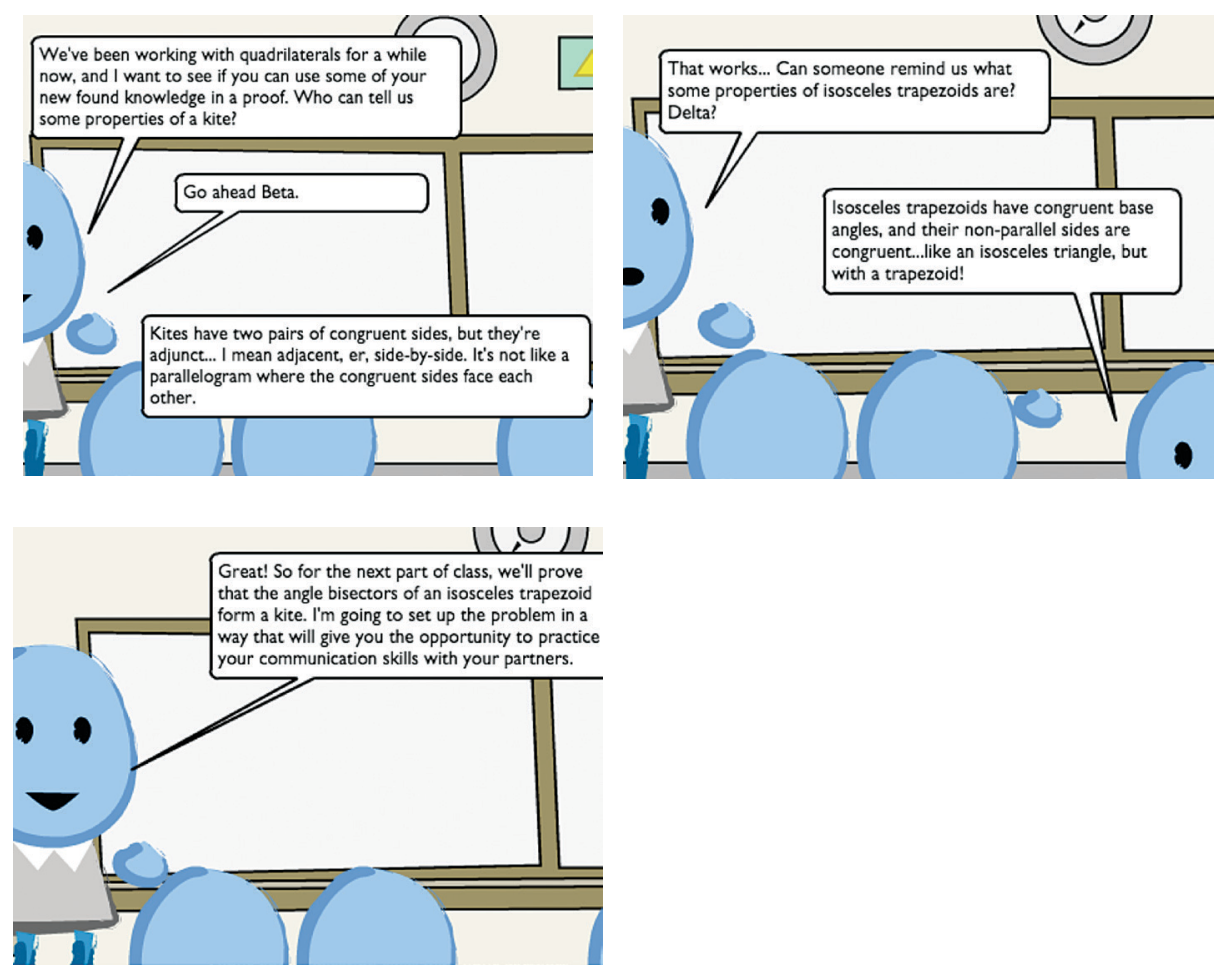

Figura 5. Lo dado para un problema de decisión ${ }^{8}$

C 2018, The Regents of the University of Michigan, used with permission

En la investigación empírica nos planteamos preguntas como, por ejemplo: ¿cuán probable es que un maestro de geometría se desvíe de la norma del

${ }^{8}$ Traducción: Cuadro 1: Maestro: Hemos estado trabajando con cuadriláteros por un tiempo. Me gustaría ver si pueden ustedes usar este conocimiento para hacer una demostración. ¿Quién puede recordarnos la definición de romboide? A ver, Beta. Beta: Los romboides tienen dos pares de lados congruentes, pero son adjuntos... quiero decir adyacentes, uno al lado del otro. No como el paralelogramo donde los lados congruentes están uno enfrente del otro.

Cuadro 2. Maestro: De acuerdo. ¿Y quién puede recordarnos las propiedades del trapecio isósceles? ¿Delta? Delta: Los trapecios isósceles tienen congruentes los ángulos en la base ... tal como en un triángulo isósceles, pero con un trapecio.

Cuadro 3. Maestro: Muy bien. Entonces durante el resto de la hora vamos a demostrar que las bisectrices de los ángulos del trapecio isósceles son los vértices de un romboide. Voy a darles el problema de manera que practiquen como comunicarse entre ustedes." 
registro diagramático cuando presenta un problema de demostración? En el caso del problema que planteamos antes, la Figura 5 muestra qué es lo que ha ocurrido en la clase inmediatamente antes de que el maestro presente el problema. Luego de mostrar esta escena les preguntamos a nuestros participantes qué harían ellos, y les damos lugar para una respuesta abierta. Luego les damos cuatro alternativas, que se muestran en la Figura 6.

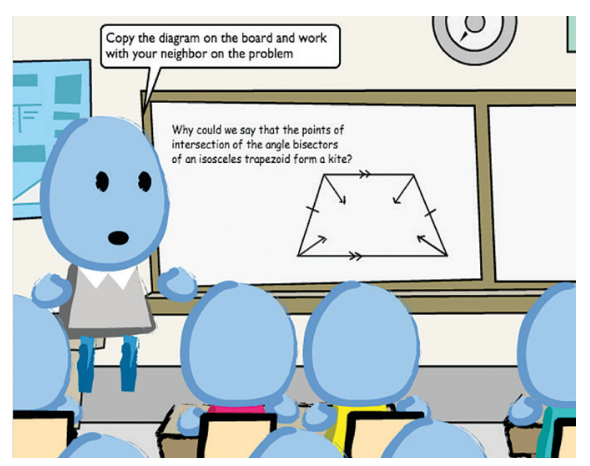

Opción A

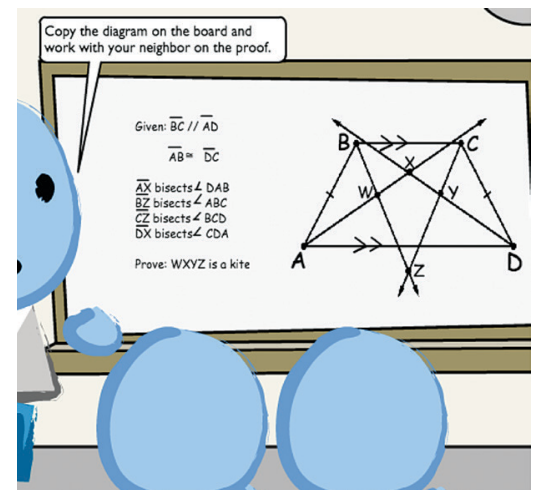

Opción C

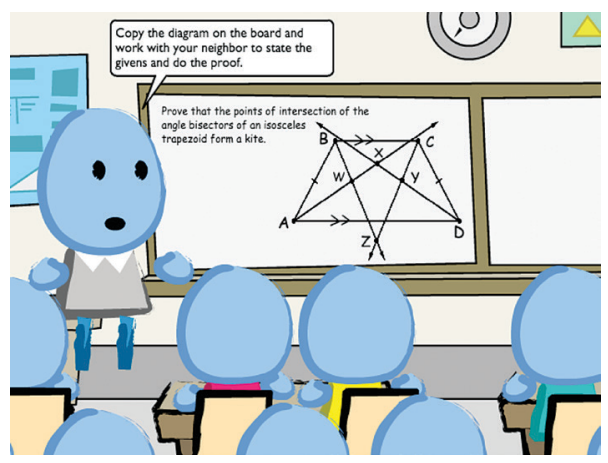

Opción B

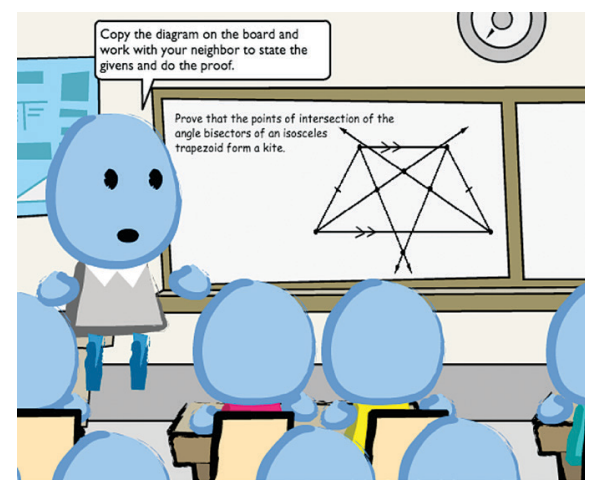

Opción D

Figura 6. Cuatro opciones para la decisión a tomar ${ }^{9}$ (ver Figura 5)

(C) 2018, The Regents of the University of Michigan, used with permission

\footnotetext{
9 Traducción. Salvo variaciones menores, en todos los casos el maestro dice "Copien el diagrama del tablero y hagan la demostración con su compañero."
} 
De acuerdo con lo que notamos más arriba, la opción C es la que se apega más a la norma del registro diagramático. Como ya mencionamos, la opción A no provee el diagrama del romboide y la opción B, si bien lo provee, enuncia el problema en términos conceptuales. La opción D, no solamente hace eso, sino que también omite las letras que identificarían los vértices. Ahora bien, no estamos tan interesados en verificar que la mayoría de los participantes elija la Opción C; estamos interesados en generar variabilidad, para que después podamos explicar esa variabilidad utilizando regresión (ver Herbst et al., 2016).

Entonces ¿cómo se utilizan los datos que generamos? Cada respuesta a cada ítem provee una respuesta categórica, eligiendo una de esas opciones. Una muestra provee porcentajes de participantes que eligieron cada categoría. Utilizamos regresión logística multinomial para estudiar como variable dependiente la probabilidad de elegir una alternativa a la norma en referencia a la probabilidad de elegir la norma. Unas cuantas variables independientes pueden intentarse como predictores de aquella probabilidad -por ejemplo-, la cantidad del conocimiento matemático de los maestros en geometría, el grado de reconocimiento de la norma, o el grado de reconocimiento de la obligación que podría justificar tal elección.

Para recapitular, nuestro objetivo es diseñar maneras confiables de medir los recursos sociotécnicos disponibles para el maestro, en este caso el reconocimiento de las normas en una situación y el reconocimiento de las obligaciones profesionales, de tal suerte que podamos usarlos para entender la toma de decisiones en situaciones de instrucción. Nos interesa crear simulaciones de la toma de decisiones en situaciones de instrucción, y dar cuenta de la variabilidad de las decisiones que toman los maestros en términos de factores tanto individuales como socio-técnicos.

\section{CONCLUSIÓN: NUESTRA METODOLOGÍA Y LA RACIONALIDAD DE LA PRÁCTICA}

El lector puede preguntarse por qué usamos los caracteres animados. Esta pregunta da lugar a una conexión metodológica importante para nosotros y que puede ser desestimada muy fácilmente. Hay gente que aún considera a los dibujos animados como una cosa de niños. Afortunadamente el mercado editorial ha generado en los últimos años una gran producción de novelas gráficas para adultos que ayuda a combatir aquel prejuicio (e.g., Doxiadis et al., 2010; Ottaviani \& Purvis, 2016). Nosotros hemos usado dibujos animados para 
representar escenas en la enseñanza de las matemáticas, para mostrárselas a maestros desde el 2004 (pero puede verse un ejemplo anterior en el apéndice del artículo de Balacheff, 1988).

Dos líneas de razonamiento apoyan nuestra apuesta a los dibujos animados. Una es que la racionalidad de la práctica y el conocimiento de la enseñanza son por lo general tácitos y los dibujos son una manera de preguntar acerca de aquéllos sin tener que comprometer una representación simbólica (por ejemplo, sin tener que comprometer enunciados hechos en un vocabulario especializado): las tiras cómicas, como los diálogos, nos permiten crear narrativas de la práctica en lugar de describirla (Zazkis \& Herbst, 2018), además las tiras cómicas nos permiten mostrar sin nombrar, lo cual es particularmente útil a la hora de estudiar el conocimiento tácito. Esto surge de la manera como conceptualizamos la racionalidad de la práctica la que, de acuerdo con Bourdieu (1998), incluye categorías de percepción y categorías de apreciación. Uno puede imaginarse que si no tuviéramos los dibujos y quisiéramos describir el enunciado de un problema de demostración a un maestro para luego hacerle una pregunta en un cuestionario, deberíamos de representar la situación y la acción usando un lenguaje elegido por el investigador, el cual no necesariamente sería el lenguaje de la práctica del maestro (pues tal lenguaje no existe -la enseñanza no tiene un vocabulario técnico que contenga las acciones matemáticamente importantes; véase Ball \& Forzani, 2011). Una representación verbal abstracta de las decisiones de enseñanza pone más en peligro, que una representación icónica, nuestra capacidad de captar cuáles son las categorías de percepción de los maestros. Los dibujos animados nos permiten mostrar y narrar la práctica en lugar de nombrarla y describirla: éstas son características deseables en una metodología que pretende obtener de los maestros mismos información acerca de su racionalidad.

La segunda línea de razonamiento es que la racionalidad de la práctica incluye no sólo las consideraciones que subtienden lo real (lo que realmente ocurre en la práctica) sino también lo viable, lo concebible, y la diferencia entre aquéllos. Los problemas de decisión, en la medida en que ellos representan decisiones que el maestro debe de tomar, nos requieren considerar lo que será elegido, contra un trasfondo de posibilidades concebibles y tal vez también viables. La representación de esas alternativas requiere un lenguaje, pero la línea de razonamiento anterior sugiere que tal lenguaje puede beneficiarse de mantener componentes icónicas e indiciales en lugar de ser completamente transpuesto a un lenguaje simbólico. Esto nos deja dos posibilidades, el lenguaje de la actuación teatral y el lenguaje de los dibujos animados. Ambos son viables, 
y cada uno de ellos tienen ventajas sobre el otro. Nosotros hemos elegido trabajar con dibujos animados en lugar de actores pues los dibujos nos permiten no solamente la facilidad de crear escenas con un costo de producción muy bajo (usando la plataforma LessonSketch; Herbst, Aaron, \& Chieu, 2013) sino también la posibilidad de pensar a la semiótica de la práctica constructivamente: en lugar de replicar todos los elementos visuales en la acción humana, cada una de las posibilidades gráficas de diferenciación (por ejemplo, las maneras de representar distintos colores de piel o expresiones faciales) no necesita darse por sentada sino que puede ser estudiada empíricamente en términos del papel que juegan en la representación de la enseñanza de las matemáticas.

\section{REFERENCIAS}

Balacheff, N. (1988). Aspects of Proof in Pupils' Practice of School Mathematics. In D. Pimm (Ed), Mathematics, teachers and children. London: Hodder Stoughton.

Ball, D. L., \& Forzani, F. M. (2011). Building a Common Core for learning to teach and connecting professional learning to practice. American Educator, 35(2), 17-21, 38-39. Ball, D. L., Thames, M. H., \& Phelps, G. (2008). Content Knowledge for Teaching what Makes it Special? Journal of Teacher Education, 59(5), 389-407.

Boileau, N., Dimmel, J.K., \& Herbst, P. G. (2016, November). Teachers' Recognition of the Diagrammatic Register and its Relationship with their Mathematical Knowledge for Teaching. In M.B. Wood, E.E. Turner, M. Civil, \& J.A. Eli (Eds.), Proceedings of the Thirty-Eighth Annual Meeting of the North American Chapter of the International Group for the Psychology of Mathematics Education. Tucson, Arizona. http://hdl.handle. net/2027.42/137972.

Boileau, N., \& Herbst, P. G. (2015, November). Teachers' expectations about Geometric Calculations in High School Geometry. In T.G. Bartell, K.N. Bieda, R.T. Putnam, K. Bradfield \& H. Dominguez (Eds.), Proceedings of the Thirty-Seventh Annual Meeting of the North American Chapter of the International Group for the Psychology of Mathematics Education East Lansing, MI. http://hdl.handle.net/2027.42/137971.

Bourdieu, P. (1990). The Logic of Practice. Stanford, CA: Stanford University Press.

Bourdieu, P. (1998). Practical Reason: On the Theory of Action. Stanford, CA: Stanford University Press.

Brousseau, G. (1997). Theory of Didactical Situations in Mathematics: Didactique des Mathematiques 1970-1990 (Ed. \& Trans. by N. Balacheff, M. Cooper, R. Sutherland, \& V.Warfield). Dordrecht, Netherlands: Kluwer. 
Bryk, A., Gomez, L., Grunow, A., \& LeMahieu, P. (2015). Learning to Improve: How America's Schools Can Get Better at Getting Better. Cambridge, MA: Harvard.

Bush, G. W. (2002). No Child Left Behind, Act of 2001, Pub. L. No. 107-110, § 115. Stat, $1425,107-110$.

Chazan, D. \& Herbst, P. (2012). Animations of Classroom Interaction: Expanding the Boundaries of Video Records of Practice. Teachers' College Record, 114(3).

Chazan, D., Herbst, P., \& Clark, L. (2016). Research on the Teaching of Mathematics: A Call to Theorize the Role of Society and Schooling in Mathematics. In D. Gitomer \& C. Bell (Eds.), Handbook of research on teaching (5th ed., pp. 1039-1097). Washington, DC: AERA.

Chevallard, Y. (1992). La transposition didactique: Du savoir savant au savoir enseignée (2 ${ }^{\text {nd }}$. ed). Grenoble: La Pensée Sauvage.

Cohen, D. K., Raudenbush, S. W., \& Ball, D. L. (2003). Resources, Instruction, and Research. Educational Evaluation and Policy Analysis, 25(2), 119-142.

Dimmel, J. \& Herbst, P. (2017). Secondary Mathematics Teachers' Expectations of Student Communication Practices when doing Proofs in Geometry. Teaching and Teacher Education, 68, 151-160.

Dimmel, J. \& Herbst, P. (2018). What Details do Teachers Expect from Students' Proofs? A study of routines for checking proofs. Journal for Research in Mathematics Education, 49(3), 261-291.

Doxiadis, A., Papadimitriou, C. H., Papadatos, A., \& Di Donna, A. (2010). Logicomix: An Epic Search for Truth. New York: Bloomsbury.

Erickson, A. \& Herbst, P. (2016, online first). Will Teachers Create Opportunities for Discussion when Teaching Proof in a Geometry Classroom? International Journal of Mathematics and Science Education.

Goffman, E. (1974). Frame Analysis: An Essay on the Organization of Experience. Boston: Northeastern University Press.

Herbst, P. (2003). Using Novel Tasks to Teach Mathematics: Three Tensions Affecting the Work of the Teacher. American Educational Research Journal, 40, 197-238.

Herbst, P. (2005). Knowing About "Equal Area” While Proving a Claim about Equal Areas. Recherches en Didactique des Mathématiques, 25, 11-56.

Herbst, P. (2006). Teaching Geometry with Problems: Negotiating Instructional Situations and Mathematical Tasks. Journal for Research in Mathematics Education, 37, 313-347.

Herbst, P., Aaron, W., \& Chieu, V. M. (2013). LessonSketch: An Environment for Teachers to Examine Mathematical Practice and Learn about its Standards. In D. Polly (Ed.), Common Core Mathematics Standards and Implementing Digital Technologies (pp. 281-294). Hershey, PA: IGl Global. 
Herbst, P. \& Chazan, D. (2012). On the Instructional Triangle and Sources of Justification for Actions in Mathematics Teaching. ZDM The International Journal of Mathematics Education, 44(5), 601-612.

Herbst, P. \& Chazan, D. (2015). Using Multimedia Scenarios Delivered Online to Study Professional Knowledge Use in Practice. International Journal of Research and Method in Education, 38(3), 272-287.

Herbst, P., Chazan, D., Kosko, K., Dimmel, J. \& Erickson, A. (2016). Using Multimedia Questionnaires to Study Influences on the Decisions Mathematics Teachers Make in Instructional Situations. ZDM The International Journal of Mathematics Education, 48, 167-183.

Herbst, P., Chen, C., Weiss, M., \& González, G., with Nachlieli, T., Hamlin, M., \& Brach, C. (2009). "Doing proofs" in Geometry Classrooms. In M. Blanton, D. Stylianou, \& E. Knuth (Eds.), Teaching and Learning of Proof across the Grades: A K-16 Perspective (pp. 250-268). New York: Routledge.

Herbst, P., Dimmel, J., Erickson, A., Ko, I., \& Kosko, K. (2014, July). Mathematics Teachers' Recognition of an Obligation to the Discipline and its Role in the Justification of Instructional Actions. In C. Nicol, P. Liljedahl, S. Oesterle, \& D. Allen (Eds.), Proceedings of the 38th annual meeting of the International Group for the Psychology of Mathematics Education and the 36th annual meeting of the North American Chapter of PME (Vol. 3, pp. 273-280). Vancouver, Canada: Simon Fraser University.

Herbst, P., \& Kosko, K. (2014a). Mathematical Knowledge for Teaching and its Specificity to High School Geometry Instruction. In J. Lo, K. R. Leatham, \& L. R. Van Zoest (Eds.), Research Trends in Mathematics Teacher Education (pp. 23-45). New York, NY: Springer.

Herbst, P. \& Kosko, K. (2014b). Using Representations of Practice to Elicit Mathematics Teachers' Tacit Knowledge of Practice: A Comparison of Responses to Animations and Videos. Journal of Mathematics Teacher Education, 17(6), 515-537.

Herbst, P., Kosko, K., \& Dimmel, J. (2013, November). How are Geometric Proof Problems Presented? Conceptualizing and Measuring Teachers' Recognition of the Diagrammatic Register. In Martinez, M. \& Castro Superfine, A (Eds.). Proceedings of the 35th annual meeting of the North American Chapter of the International Group for the Psychology of Mathematics Education (pp. 179-186). Chicago, IL: University of Illinois at Chicago.

Herbst, P., Nachlieli, T., \& Chazan, D. (2011). Studying the Practical Rationality of Mathematics Teaching: What Goes into "Installing" a Theorem in Geometry? Cognition and Instruction, 29(2), 218-255. 
Hsu, H. Y., \& Silver, E. A. (2014). Cognitive Complexity of Mathematics Instructional Tasks in a Taiwanese Classroom: An Examination of Task Sources. Journal for Research in Mathematics Education, 45(4), 460-496.

Kilpatrick, J. (1990). Review: Apples and Oranges Again: Reviewed Works: The IEA Study of Mathematics I: Analysis of Mathematics Curricula by Kenneth J. Travers \& lan Westbury; The IEA Study of Mathematics II: Contexts and Outcomes of School Mathematics by David F. Robitaille \& Robert A. Garden. Journal for Research in Mathematics Education, 21(5), 416-424.1.

Manders, K. (2008). The Euclidean Diagram. In: Mancosu, P. (Ed.) The Philosophy of Mathematical Practice (pp. 80-133). Oxford: OUP. (Original work dated 1995).

Mehan, H., \& Wood, H. (1975). The Reality of Ethnomethodology. Malabar, FL; Krieger.

Nachlieli, T. \& Herbst, P. with González, G. (2009). Seeing a Colleague Encourage a Student to Make an Assumption While Proving: What Teachers Put to Play in Casting an Episode of Geometry Instruction. Journal for Research in Mathematics Education, 40(4), 427-459.

Ottaviani, J. \& Purvis, L. (2016). The Imitation Game: Alan Turing decoded. New York: Abrams.

Weiss, M., Herbst, P., \& Chen, C. (2009). Teachers' Perspectives on "Authentic Mathematics" and the Two-column Proof Form. Educational Studies in Mathematics, 70 (3), 275-293.

Zazkis, R. \& Herbst, P. (Eds, 2018). Scripting Approaches in Mathematics Education: Mathematical Dialogues in Research and Practice. New York: Springer. 